\title{
Conventional and emerging treatments in the management of acute primary angle closure
}

This article was published in the following Dove Press journal:

Clinical Ophthalmology

14 March 2012

Number of times this article has been viewed

\section{Pui Yi Boey'}

\section{Shweta Singhal'}

Shamira A Perera'

Tin Aung ${ }^{1,2}$

'Singapore Eye Research Institute, Singapore National Eye Centre,

${ }^{2}$ Department of Ophthalmology, Yong Loo Lin School of Medicine, National University of Singapore, Singapore
Correspondence: Tin Aung Singapore National Eye Centre, II Third Hospital Avenue, Singapore 16875 I

Tel +6562277255

Fax +6562263395

Email tin I I@pacific.net.sg
Abstract: The management of acute primary angle closure is directed at lowering the intraocular pressure and relieving pupil block. Conventional treatment involves the use of medical treatment and laser peripheral iridotomy, respectively, as a means for achieving these aims. Newer therapeutic strategies have been described that are potentially useful adjuncts or alternatives to conventional treatment. Emerging strategies that lower intraocular pressure include anterior chamber paracentesis, as well as laser procedures such as iridoplasty and pupilloplasty. A possible alternative to relieving pupil block is lens extraction, and may be combined with adjunctive measures such as goniosynechiolysis and viscogoniosynechiolysis. Trabeculectomy has a limited role in the acute setting. This review paper reviews the current evidence regarding conventional and newer treatment modalities for acute primary angle closure.

Keywords: intraocular pressure, pupil block, anterior chamber paracentesis, iridoplasty, pupilloplasty, goniosynechiolysis, viscogoniosynechiolysis, trabeculectomy

\section{Introduction}

Acute primary angle closure (APAC) is an important cause of irreversible visual loss, particularly in East Asians in whom there is an incidence of 12.2 cases of APAC per 100,000 per year reported in Singapore. The incidence is higher among Chinese people than in Malay or Indian people, in females, and those aged $\geq 60$ years..$^{1-3}$ APAC has serious long-term consequences and is potentially blinding. The timely management of an acute attack of primary angle closure (PAC) is important not only for preventing visual loss from the high-pressure episode but also for preventing progression to chronic angle closure glaucoma (CACG). A retrospective study has shown that, following resolution of an episode of APAC, 58.1\% patients have raised intraocular pressure (IOP) requiring ocular hypotensive medications, with $32.7 \%$ eventually requiring trabeculectomy. ${ }^{4}$ In the 4-10 years following an APAC attack, Aung et al reported that $18 \%$ of eyes had become blind, $48 \%$ of eyes had developed glaucomatous optic neuropathy, and $58 \%$ of eyes had vision worse than $20 / 40.5$

The conventional practice in the management of APAC is to initially lower the IOP with topical and systemic medical therapy. This allows resolution of corneal edema and secondary inflammation, thereby enabling subsequent definitive treatment of the condition by relieving pupil block typically using laser peripheral iridotomy (LPI). ${ }^{6,7}$ The goals of treatment are to lower IOP as quickly as possible in order to alleviate the suffering of the patient, reduce the risk of irreversible damage to the optic nerve head and other anterior segment structures, to prevent recurrent attacks and retard progression to $\mathrm{CACG}^{8}$ 
In this review paper, both the conventional and newer options in the management of APAC are discussed, with emphasis on methods of lowering IOP, techniques to relieve pupil block, and other adjunctive measures that may be useful alternatives in this condition.

\section{Lowering IOP to allow resolution of the corneal edema Conventional management} Medical treatment

Conventional medical treatment of IOP includes systemic medication such as hyperosmotic agents and carbonic anhydrase inhibitors as first-line treatment and various combinations of IOP-lowering agents, including topical beta-blockers, prostaglandin analogues, alpha-adrenergic agonists, and carbonic anhydrase inhibitors. Topical steroids are usually added to control intraocular inflammation. Once the IOP is lowered, pilocarpine can be used to constrict the pupil from its mid-dilated state and help pull the iris away from the iridocorneal angle.

In one study, the use of medical therapy alone (comprising a combination of intravenous acetazolamide followed by oral acetazolamide, as well as topical pilocarpine, timolol, and steroid drops) successfully aborted the episode of APAC within the first 24 hours in $89.2 \%$ of patients. ${ }^{9}$ However, this success rate is lower at preceding time points: $21.5 \%$ at 3 hours, $44.6 \%$ at 6 hours, and $76.2 \%$ at 12 hours. Given this slow rate of resolution, it has been argued that other techniques like anterior chamber paracentesis and argon laser peripheral iridoplasty (ALPI) may cause less damage to the optic nerve and other anterior segment structures by having a more rapid onset of action and quicker IOP reduction. Complications from medical therapy, though rare, can be potentially serious, such as metabolic acidosis and electrolyte disorders with acetazolamide ${ }^{10,11}$ and congestive cardiac failure with mannitol. ${ }^{7,9,12,13}$ Fortunately, the incidence of these complications is low and medical therapy still forms the mainstay of first-line therapy for APAC given its relative safety and efficacy.

\section{Alternative strategies}

\section{Anterior chamber paracentesis}

Anterior chamber (AC) paracentesis is an alternative method of lowering IOP in APAC and involves controlled drainage of aqueous from the anterior chamber by the use of a needle or incision with a blade. This can be performed using a slit lamp with the patient under topical anesthesia and aseptic conditions. AC paracentesis has the advantages of rapidly lowering IOP and being repeatable. ${ }^{7}$ With IOP lowered to adequate levels (albeit temporarily), corneal edema may clear enough to allow for earlier definitive treatment of pupil block with LPI.

The literature on this treatment modality is currently limited and mainly consists of descriptive case reports. This is possibly due to various technical difficulties, which will be elaborated upon further in this paper, as well as availability of other established effective treatments. A case series of ten eyes showed that AC paracentesis lowered IOP more rapidly compared with eyes treated with conventional medical therapy, in which mean IOP dropped immediately from $66.6 \mathrm{mmHg}$ preparacentesis to $15.1 \mathrm{mmHg}$ and $21.7 \mathrm{mmHg}$ at 30 minutes postparacentesis. ${ }^{14}$ This was in comparison with the medication-only group, where mean IOP was $62.3 \mathrm{mmHg}$ before treatment, dropping to $41.5 \mathrm{mmHg}, 30$ minutes after treatment. After 2 hours the IOP remained $<21 \mathrm{mmHg}$ (comparable to eyes treated medically), although, beyond 2 hours after paracentesis, IOP gradually drifted upward to levels comparable to the medication-only group; this is not unexpected since the procedure does not relieve pupil block. Hence, the benefits of AC paracentesis are short-lived and repeat treatment may be required. It may be best considered a temporizing measure until definitive treatment is instituted. Another case series looking at AC paracentesis used in conjunction with medical treatment for 14 cases of APAC and six cases of secondary glaucoma, found that IOP decreased from $53.4 \mathrm{mmHg}$ preprocedure to $18.2 \mathrm{mmHg}$ at 24 hours. ${ }^{15}$ Relief of symptoms was seen in all patients, while corneal edema cleared in 17 cases; the three cases that failed came from the secondary glaucoma group. The authors reported no complications from the procedure.

An AC paracentesis must be undertaken with caution. ${ }^{14}$ The procedure may be technically challenging, due to both patient and ocular factors. The patient may be in great discomfort with nausea or vomiting, and may not be able to cooperate with the procedure. Some, therefore, recommend performing the procedure under peribulbar local anesthesia. The AC may be extremely shallow, presenting the risk of damage to the surrounding iris, lens, or corneal endothelium. Malignant glaucoma may also result, due to rapid forward movement of the iris-lens diaphragm. Rapid decompression can precipitate a suprachoroidal hemorrhage or more commonly a decompression retinopathy or hyphema. Last but not least, as this is an intraocular procedure, the risk of endophthalmitis will always be present. Currently, this remains as an approach for patients for whom medical therapy is either unavailable or unsuitable, or for those that are approaching their maximal dosages for certain systemic 
medications and still have unresolved APAC. If this fails to yield a clear cornea for LPI, then the next option would be to perform a surgical iridectomy.

\section{ALPI and argon laser pupilloplasty}

ALPI has been advocated in the management of APAC and has the weight of randomized controlled trials to support its use. It involves applying laser burns to the peripheral iris, causing contraction of the iris stroma and mechanically pulling the angle open, hence facilitating trabecular aqueous drainage. ${ }^{7,16-18}$ ALPI has been reported to be a safe and effective alternative to medical treatment of APAC in a prospective randomized controlled trial. ${ }^{16}$ After initial treatment of topical timolol and pilocarpine, patients in this trial were randomized to either ALPI or medical treatment, which comprised systemic acetazolamide, as well as mannitol if the initial IOP was $>60 \mathrm{mmHg}$. The study found that ALPI achieved lower IOPs at 15, 30, and 60 minutes after the institution of treatment. The duration of symptoms before treatment did not affect the efficacy of ALPI and, in long-term follow-up, there were no differences in mean IOP, the requirement for IOP-lowering agents, or the extent of peripheral anterior synechiae (PAS) between both groups. By changing the profile of the peripheral iris, ALPI may also remove a component of ciliary block. ${ }^{19}$

Another randomized controlled trial compared LPI alone to LPI with peripheral iridoplasty in patients with PAC and PAC glaucoma. ${ }^{18}$ The extent of PAS decreased significantly more in the combined LPI and iridoplasty group, compared with the LPI-alone group. There was no difference in IOP, need for medications and surgery, and visual function at 1 year. As this study did not include patients who presented with APAC, these results may not be directly extrapolatable to our group of interest. Most of the studies on ALPI have been done on Asian eyes and ALPI is not widely used elsewhere. This may be a reflection of the populations in which APAC is most common or the lack of evidence of its use in nonbrown irides.

ALPI offers the advantage of more rapid reduction in the IOP in the first few hours of the episode compared with conventional medical therapy. Further, it has been shown to reduce the formation of PAS (likely by cutting down the duration of appositional angle closure), thereby retarding the progression to CACG. ${ }^{16}$ In current practice, ALPI may not be widely accepted due to various difficulties associated with its use. Corneal clouding may pose technical difficulties. Also, there is a learning curve in the technique of performing ALPI, and as yet there are many different ways of performing this. Another possible limitation, based on experience at our centre, is that APAC patients are often seen after office hours by more junior doctors who may not be sufficiently skilled in performing this procedure. There are no ideal laser parameters or number of spots required, and there is little evidence of its efficacy in eyes with significant PAS. ${ }^{20}$

Argon laser pupilloplasty is another technique that is useful in APAC where corneal clouding precludes LPI, and this may be used alone or in combination with other procedures such as ALPI to abort an acute attack. ${ }^{21}$ In this procedure, radial rows of laser contraction burns are applied to the pupillary border to cause pupil dilation. The burns may be performed around the entire circumference of the pupil to cause symmetrical dilation, or in a quadrant for focal dilation. Like ALPI, this is a temporizing measure to allow corneal edema to clear more quickly prior to LPI.

\section{Corneal indentation $(\mathrm{Cl})$}

Indentation on the cornea can mechanically open the angle, forcing aqueous through the trabecular meshwork, thereby reducing IOP in APAC. It is a quick and readily available way of reducing IOP in the acute setting, particularly in situations where there is no access to medications or laser. ${ }^{22}$ Various tools have been used for corneal indentation, including muscle hooks, gonioprism lenses, cotton-tip applicators, and glass rods. Corneal indentation offers a controlled clearance of corneal edema before the relief of pupil block is attempted. The effect is short-lived, but the procedure is easy to perform, does not require expensive or complicated equipment, and is usually (though not always) effective.

The procedure itself is not standardized. Small case series describing the use and effect of corneal indentation have shown that most cases of APAC (presenting early and without PAS formation) respond well to corneal indentation after a short course of treatment (3-4 cycles of 30 seconds of pressure on, followed by pressure off) with any of the corneal indentation tools previously mentioned. ${ }^{23,24}$ The response to corneal indentation seems independent of presenting IOP. Masselos et $\mathrm{al}^{23}$ reported a case series of seven patients with six responding effectively to $\mathrm{CI}$ (three of whom were treated with indentation following failed medical therapy), while Teichman ${ }^{24}$ reported a case series of five patients studied over 8 months where corneal indentation was successful.

When performing indentation, it has been suggested that if using cotton-tip applicator/glass rod, the endpoint of indentation is reached when the pupil margin moves. When using a gonioprism like the Zeiss 4 mirror lens (Carl Zeiss, Chester, VA), the endpoint is identified when the trabecular meshwork 
is visualized postindentation. Knowledge of these endpoints will prevent excessive pressure being applied to the cornea. It is recommended that indentation be performed over the inferior cornea to prevent damage to superior corneal epithelium, thereby facilitating subsequent LPI. Masselos et al also found a possible association between the success of CI and LPI. Patients who failed to respond to CI with reduction in IOP were found to be refractory to subsequent LPI as well and it is postulated that CI may have a further role in predicting the effectiveness of LPI in patients, thus aiding in decision making regarding further treatment. ${ }^{23}$

There have been some concerns raised regarding the effect of raised IOP during the process of indentation causing ischemic damage to ocular tissues. ${ }^{23}$ However, the transient IOP increase during indentation has been found to be insufficient to cause permanent vascular disruption to the retina or optic nerve and no cases of optic nerve head infarction or retinal vascular occlusion were seen in the case series reported. ${ }^{23}$ Corneal indentation can also be used in conjunction with any of the other procedures used for APAC.

\section{Supine positioning of the patient}

Lying the patient supine is recommended as it allows the lensiris diaphragm to move posteriorly with gravity, which may relieve some of the relative pupil block. This is particularly useful in such instances where there are multiple mechanisms involved and where there is zonular instability.

\section{Corneal scraping}

The edematous corneal epithelium, which may preclude a LPI, can be scraped off with a needle under topical anesthesia to create an area of clear cornea through which a LPI may be attempted.

\section{Relieving pupil block}

Once the IOP has been lowered sufficiently to allow corneal edema to clear and reduce the risk of ischemic damage to the optic nerve, definitive treatment to prevent recurrence of another acute attack must be performed. Definitive treatment involves overcoming the block at the level of the pupil, preventing aqueous from flowing from the posterior chamber to the anterior chamber. This is most commonly done by creating a conduit through the iris stroma either surgically (surgical iridectomy) or, more commonly, using a laser (LPI). In some cases removal of the lens can allow sufficient deepening of the anterior chamber to overcome the block. The fellow eye of APAC also requires prophylactic treatment with a laser PI, since half of these will otherwise suffer an acute attack within 5 years. ${ }^{6}$

\section{Conventional treatment} LPI

In the past, surgical iridectomy was performed for APAC, ${ }^{25-28}$ though in recent years LPI has become the treatment of choice, due to its non-invasive nature, ease of performing the procedure on an outpatient basis, and the low risk of complications. ${ }^{6}$

While Caucasian eyes usually benefit from LPI performed using neodymium-doped yttrium aluminium garnet (Nd:YAG) lasers alone, heavily pigmented irides have been shown to benefit from sequential argon laser and Nd:YAG laser which allows effective PI formation while minimizing tissue damage to the iris. ${ }^{29-31}$ In Caucasian eyes treated for APAC with LPI/surgical iridectomy, iridotomy alone is sufficient to control IOP $65 \%-76 \%$ of the time, and additional medical therapy can control the IOP in up to $84 \%-$ $99 \%$ of eyes. Furthermore, a prophylactic LPI is particularly effective in almost completely preventing an APAC attack in the fellow eye. ${ }^{32,33}$ In Asian eyes however, use of LPI alone does not guarantee good IOP control and a subsequent rise in IOP is seen in $76.6 \%$ of eyes within the first 6 months of the acute attack. Of all Asian eyes treated for APAC with LPI, $58.2 \%$ still require the use of additional antiglaucoma medication/filtration surgery to control IOP. ${ }^{32,33}$

Some cases of APAC may be refractory to treatment with LPI and the attack may remain unbroken. Cases where pupil block is not the mechanism of angle closure may not respond as well and still progress despite conventional treatment (for example, plateau iris). ${ }^{34}$ Performing LPI is still indicated in these cases as there may still be a component of pupil block. LPI may break some fine PAS, but the more established areas of PAS usually remain even after successful LPI despite the resultant angle widening. If the IOP remains persistently high, the formation of PAS or ultrastructural damage to the trabecular meshwork results in a compromised angle, which would predispose to CACG.

Although usually quite safe, LPI can occasionally be associated with complications including monocular diplopia, visual aberrations, bleeding/hyphema, IOP spikes, closure of the peripheral iridotomy due to pigmentary proliferation and corneal decompensation. ${ }^{35-38}$

\section{Alternative strategies}

\section{Lens extraction}

Primary lens extraction as treatment for APAC has been reported in many studies and can be considered as another modality in management. ${ }^{39-46}$ Lens extraction in APAC relieves pupil block and markedly decreases angle crowding, thus widening the angle. ${ }^{39,40}$ Ultrasound biomicroscopy shows that 
cataract extraction results in angle widening and resolution of anterior-placed ciliary processes in PAC eyes. ${ }^{41}$

In cases of APAC where IOP is uncontrolled, lens extraction has been shown to result in good IOP control without medications in $67 \%-72 \%$ of cases. ${ }^{39}$ The greatest IOP reduction after lens extraction was seen in APAC eyes where IOP was uncontrolled preoperatively, compared with lens extraction performed for other non-acute angle closure eyes. ${ }^{42,43}$ A small case series of primary lens extraction for APAC examined the short-term effects on IOP. ${ }^{44}$ The authors of the series demonstrated a $9.0 \mathrm{mmHg}$ drop in mean IOP on the first postoperative day and a $10.2 \mathrm{mmHg}$ drop after the first postoperative week, compared with preoperative IOP of $22.8 \mathrm{mmHg}$. Other studies report IOP results in the longer term. Jacobi et al, for example, compared primary phacoemulsification and surgical iridectomy for treatment of APAC eyes and reported that glaucoma control was achieved in a significantly larger proportion in the phacoemulsification group ( $72 \%$ vs 35\% respectively) and a lower proportion of this group required additional surgery (11.5\% vs 63\%). ${ }^{45}$ Lam et al described the role of early phacoemulsification for APAC in a randomized controlled trial, where it was found that eyes undergoing LPI were 15 times more likely to have an IOP rise above $21 \mathrm{mmHg}$ compared with eyes that had early phacoemulsification. ${ }^{46}$ At 18 months, the LPI group required more medications to control IOP compared with the phacoemulsification group.

However, lens extraction in an acute setting of APAC may pose technical difficulties during surgery (due to shallow anterior chamber depth, small pupils and/or floppy iris, intumescent or subluxed lenses, and cornea edema) and postoperative complications such as corneal decompensation. ${ }^{40}$ Its popularity in APAC management has followed the technological advances in small-incision cataract surgery and the increasing expertise amongst the surgeons, making this option much more viable in recent times. Another consideration is the timing of lens extraction in an acute setting, weighing the need for acute IOP lowering as soon as possible against surgery in an inflamed edematous eye. ${ }^{40}$ Topical anesthesia may not be adequate in more inflamed eyes and there is the concern that surgery on an inflamed eye may result in more postoperative complications. These controversies are as yet unresolved and the optimum timing for lens extraction is unknown.

\section{Adjunctive measures}

While a significant number of cases of APAC respond well to medical therapy and subsequent LPI, presence of extensive PAS (usually more than $270^{\circ}$ of closed angle) has a considerable negative impact on the success of this treatment modality and surgical intervention may be required. ${ }^{47}$ Traditionally, the failure of standard therapy was addressed with filtration surgery (trabeculectomy), possibly with combined lens extraction if warranted. ${ }^{48}$ While lens extraction alone has been shown to have some merit in treatment of unresponsive APAC patients, ${ }^{44,45}$ the benefits of addressing the PAS directly by performing synechiolysis, either independently or in combination with cataract surgery before considering filtration surgery, have recently been brought to light.

\section{Phacoemulsification of lens with intraocular lens (IOL) implantation}

As already described, phacoemulsification and IOL implantation alone can be used to treat unresponsive APAC. In many cases, the procedure deepens the anterior chamber significantly and helps in breaking pupil block. ${ }^{44,45,49}$ In addition, it has been shown that the surgical procedure itself as well as the use of viscoelastic to deepen the anterior chamber intraoperatively can cause breakdown of PAS to a certain extent (PAS $>270^{\circ}$, reduced from $43 \%$ preoperatively to $24 \%$ postoperatively).$^{50} \mathrm{In}$ this same study, IOP decreased significantly from $19.7 \mathrm{mmHg}$ preoperatively to $15.5 \mathrm{mmHg}$ postoperatively. Treatment directed at breaking PAS was therefore possibly thought to be a useful way of treating refractory APAC.

\section{Phacoemulsification with goniosynechiolysis}

Goniosynechiolysis, a surgical procedure where the PAS are deliberately broken, has been reported to be effective for IOP control and reduction of synechiae in angle closure glaucoma, although to date there is limited literature on its use in the acute setting. ${ }^{51,52}$ It involves the physical stripping of PAS from the angle wall, thus restoring aqueous access to the trabecular meshwork. It is usually effective in patients with PAS present for $<6-12$ months ${ }^{51}$ It may be performed using surgical instruments or an Nd:YAG laser. ${ }^{51,52}$ Occasionally, gonioplasty using an argon laser is used in addition to the goniosynechiolysis in cases of extremely narrow angles. ${ }^{53}$

Goniosynechiolysis is effective when performed along or in conjunction with other surgical procedures. More commonly it is performed surgically in combination with phacoemulsification. ${ }^{52,54-57}$ After phacoemulsification, the anterior chamber is deepened with viscoelastic. The goniosynechiolysis can then be performed using a blunt Swan knife by applying posterior pressure to the angle structures under direct visualization with a goniolens; the end point being 
revisualization of the trabecular meshwork. For eyes with previous APAC and PAS, goniosynechiolysis combined with cataract extraction has been found to be more effective than goniosynechiolysis alone. ${ }^{54}$ In a prospective noncontrolled clinical trial in 52 eyes of 48 patients with APAC unresponsive to LPI/ALPI, Teekhasaenee and Ritch reported 90.4\% success (mean IOP $<20 \mathrm{mmHg}$ without need for additional medication) and mean reduction of PAS from $310^{\circ}$ to $60^{\circ}$ with no recurrence of PAS up to 6 years. ${ }^{54}$ Harasymowycz et al performed a similar study using phacoemulsification with goniosynechiolysis with 21 patients who had acute or subacute angle closure glaucoma and showed a mean decline in IOP from $40.7 \mathrm{mmHg}$ with 3.8 medications preoperatively to $15.5 \mathrm{mmHg}$ with 1.7 medications over a mean follow-up of almost 1 year. ${ }^{55}$ After goniosynechiolysis, argon or diode laser peripheral iridoplasty can be used to further flatten the peripheral iris and prevent PAS reattachment. ${ }^{57,58}$

Complications of combined phacoemulsification with surgical goniosynechiolysis include fibrinoid anterior chamber reaction, photophobia, transient elevation of IOP (to $>25 \mathrm{mmHg}$ ) and hyphema. Intraoperatively, there can also be mild to severe hemorrhage from the iris or trabeculum as well as iridodialysis. ${ }^{54,56}$

\section{Phacoemulsification with viscogoniosynechiolysis}

A further modification of phacoemulsification is the replacement of the surgical instrument by viscoelastic to perform the synechiolysis, which is less traumatic and results in fewer complications. Varma et al described the use of viscogonioplasty as adjunctive treatment after phacoemulsification, using viscoelastic injected around the angles to break PAS. ${ }^{47}$ In this case series of 15 patients, at 6 months after surgery, mean IOP decreased from $52.1 \mathrm{mmHg}$ to $14.1 \mathrm{mmHg}, 14 / 15$ were free of glaucoma medications, and the trabecular meshwork was exposed over $360^{\circ}$ in all eyes with no residual synechiae. ${ }^{47}$

Razeghinejad et al described another case series of eleven APAC patients poorly responsive to medication and Nd:YAG laser iridotomy with up to $270^{\circ}$ of PAS on indentation gonioscopy who were treated with phacoemulsification, IOL implantation, and viscosynechiolysis. ${ }^{59}$ They injected viscodispersive viscoelastic (hydroxypropyl methylcellulose $2 \%$ ) near the angle after IOL implantation to divide the synechiae without contact with adjacent angle or iris. Mean follow-up of 7-8 months showed dramatic decline in mean IOP (down from $39.4 \pm 10.8 \mathrm{mmHg}$ to a mean of $13.4 \pm 2.9 \mathrm{mmHg}$ ), reduced need for additional antiglaucoma medication ( $3.8 \pm 0.7$ preoperatively to $0.4 \pm 0.9)$, and none of the patients required further filtration surgery. PAS resolved in all but one patient postsurgery. Importantly, the intraoperative and postoperative complications - for example, hemorrhage, iridocyclodialysis, and so forth - normally described with surgical goniosynechiolysis were not observed in this series, which suggests that this procedure is safer. However, critics may argue that simple viscoelastic is not strong enough to remove the more established, adhesive segments of PAS.

\section{Trabeculectomy/phacotrabeculectomy}

Filtration surgery in the form of trabeculectomy alone or combined cataract extraction is occasionally used as the final step in the management of APAC, when the condition has been refractory to all other modalities of treatment. Primary trabeculectomy early in APAC has several associated complications given the hot and inflamed nature of the eye being operated upon, including shallow or flat anterior chamber, malignant glaucoma, suprachoroidal hemorrhage, and endophthalmitis. ${ }^{60}$

In a study of trabeculectomy in patients with medically unresponsive APAC, success (defined as reducing IOP to $<22 \mathrm{mmHg}$ ) was as low as $56.2 \%$ compared with a success rate of $70 \%-95 \%$ when trabeculectomy is used conventionally for treatment of a quiet eye with CACG. ${ }^{61}$ Eyes with APAC that responded to medical treatment but had other signs suggesting CACG showed a better response to trabeculectomy with decline in IOP in about $87.5 \%$ of cases. In this study, these eyes did not receive LPI prior to the trabeculectomy. There are still insufficient data to determine whether an eye with APAC and signs of CACG is better treated with primary LPI or a primary trabeculectomy. The indication for performing trabeculectomy surgery may include those that had an acute on chronic type of presentation, where there was already extensive damage to the optic nerve and/or the persistence of high IOP after other treatments such as LPI.

The possible complications of trabeculectomy are numerous and can be sight threatening with serious consequences, which include postoperative cataract progression, hypotony with resultant maculopathy, bleb leak, blebitis, and endophthalmitis. ${ }^{62,63}$ The use of adjunctive antifibrotic agents such as mitomycin $\mathrm{C}$ may lead to increased risk of complications, including endophthalmitis, overfiltration, and hypotony. ${ }^{64-68}$

\section{Conclusion}

The conventional management of APAC involves systemic and topical medical treatment followed by LPI, though newer 
methods in the form of ALPI and primary lens extraction may offer some benefits, especially where medical treatment is inadequate or unsuitable. Other methods of breaking the initial attack, such as AC paracentesis and corneal indentation, merit some thought as they provide simple, quick, and effective alternatives to medical therapy, particularly in primary care settings. Patients with APAC refractive to medical therapy/LPI and those who have other signs of CACG and extensive PAS may require more extensive therapy. Breaking the PAS by goniosynechiolysis and viscogonioplasty in isolation or in combination with cataract extraction are useful adjunctive measures. Filtration surgery still remains the final resort and may be useful as the primary surgical intervention in some patients with signs of advanced acute on chronic CACG, but further studies are needed to determine their usefulness as a primary procedure.

While there exist an armamentarium of treatment strategies for the management of APAC, the conventional treatment of medications and LPI has a track record of efficacy. Other modalities may be considered where conventional treatment fails. Further research is required to evaluate the different management strategies for this condition which is potentially blinding.

\section{Disclosure}

The authors do not have any disclosures or conflicts of interest to declare in relation to this paper.

\section{References}

1. Congdon N, Wang, F, Tielsch, JM. Issues in the epidemiology and population-based screening of primary angle-closure glaucoma. Surv Ophthalmol. 1992;36(6):411-423.

2. Foster PJ, Aung T, Nolan WP, et al. Defining "occludable" angles in population surveys: drainage angle width, peripheral anterior synechiae, and glaucomatous optic neuropathy in east Asian people. Br J Ophthalmol. 2004;88(4):486-490.

3. Seah SK, Foster, PJ, Chew PT, et al. Incidence of acute primary angleclosure glaucoma in Singapore. An island-wide survey. Arch Ophthalmol. 1997;115(11):1436-1440.

4. Aung T, Ang LP, Chan SP, Chew PT. Acute primary angle-closure: long-term intraocular pressure outcome in Asian eyes. Am J Ophthalmol. 2001;131(1):7-12.

5. Aung T, Friedman DS, Chew PT, et al. Long-term outcomes in Asians after acute primary angle closure. Ophthalmology. 2004;111(8): 1464-1469.

6. Ang LP, Aung T, Chew PT. Acute primary angle closure in an Asian population: long-term outcome of the fellow eye after prophylactic laser peripheral iridotomy. Ophthalmology. 2000;107(11):2092-2096.

7. Lam DS, Tham CC, Lai JS, Leung DY. Current approaches to the management of acute primary angle closure. Curr Opin Ophthalmol. 2007;18(2):146-151.

8. David R, Tessler Z, Yassur, Y. Long-term outcome of primary acute angle-closure glaucoma. Br J Ophthalmol. 1985;69(4):261-262.

9. Ramli N, Chai SM, Tan GS, et al. Efficacy of medical therapy in the initial management of acute primary angle closure in Asians. Eye (Lond). 2010;24(10):1599-1602.
10. Chapron DJ, Gomolin IH, Sweeney KR. Acetazolamide blood concentrations are excessive in the elderly: propensity for acidosis and relationship to renal function. J Clin Pharmacol. 1989;29(4): 348-353.

11. Cowan RA, Hartnell GG, Lowdell CP, Baird IM, Leak AM. Metabolic acidosis induced by carbonic anhydrase inhibitors and salicylates in patients with normal renal function. Br Med J (Clin Res Ed). 1984; 289(6441):347-348.

12. D'Alena P, Ferguson, W. Adverse effects after glycerol orally and mannitol parenterally. Arch Ophthalmol. 1966;75(2):201-203.

13. Spaeth GL, Spaeth EB, Spaeth PG, Lucier AC. Anaphylactic reaction to mannitol. Arch Ophthalmol. 1967;78(5):583-584.

14. Lam DS, Chua JK, Tham CC, Lai JS. Efficacy and safety of immediate anterior chamber paracentesis in the treatment of acute primary angle-closure glaucoma: a pilot study. Ophthalmology. 2002;109(1): 64-70.

15. Arnavielle S, Creuzot-Garcher C, Bron AM. Anterior chamber paracentesis in patients with acute elevation of intraocular pressure. Graefes Arch Clin Exp Ophthalmol. 2007;245(3):345-350.

16. Lam DS, Lai JS, Tham CC, Chua JK, Poon AS. Argon laser peripheral iridoplasty versus conventional systemic medical therapy in treatment of acute primary angle-closure glaucoma: a prospective, randomized, controlled trial. Ophthalmol. 2002;109(9):1591-1596.

17. Ritch R, Tham CC, Lam DS. Argon laser peripheral iridoplasty (ALPI): an update. Surv Ophthalmol. 2007;52(3):279-288.

18. Sun X, Liang YB, Wang NL, et al. Laser peripheral iridotomy with and without iridoplasty for primary angle-closure glaucoma: 1-year results of a randomized pilot study. Am J Ophthalmol. 2010;150(1): 68-73.

19. Peng D, Zhang X, Yu K. Argon laser peripheral iridoplasty and laser iridectomy for plateau iris glaucoma. Zhonghua Yan Ke Za Zhi. 1997; 33(3):165-168. Chinese.

20. Ritch R, Liebmann JM. Argon laser peripheral iridoplasty. Ophthalmic Surg Lasers. 1996;27(4):289-300.

21. Ritch R. Argon laser treatment for medically unresponsive attacks of angle-closure glaucoma. Am J Ophthalmol. 1982;94(2):197-204.

22. Anderson DR. Corneal indentation to relieve acute angle-closure glaucoma. Am J Ophthalmol. 1979;88(6):1091-1093.

23. Masselos K, Bank A, Francis IC, Stapleton F. Corneal indentation in the early management of acute angle closure. Ophthalmology. 2009; 116(1):25-29.

24. Teichmann KD. Corneal indentation to relieve glaucoma. Am $J$ Ophthalmol. 1980;90(3):434-435.

25. Barkan O. Iridectomy in narrow-angle glaucoma. Am J Ophthalmol. 1954;37(4):504-519.

26. Barkan O. Peripheral iridectomy; technique, mechanism and the cause of hemorrhage. Am J Ophthalmol. 1954;37(6):889-896.

27. Lowe RF. Primary angle-closure glaucoma. A review 5 years after bilateral surgery. Br J Ophthalmol. 1973;57(7):457-463.

28. Playfair JT, Watson, PG. Management of acute primary angleclosure glaucoma: a long-term follow-up of the results of peripheral iridectomy used as an initial procedure. Br J Ophthalmol. 1979;63(1): $17-22$.

29. Tomey KF, Traverso CE, Shammas IV. Neodymium-YAG laser iridotomy in the treatment and prevention of angle closure glaucoma. Arch Ophthalmol. 1987;105(4):476-481.

30. Ang LP, Ang LP. Current understanding of the treatment and outcome of acute primary angle-closure glaucoma: an Asian perspective. Ann Acad Med Singapore. 2008;37(3):210-215.

31. Ho T, Fan R. Sequential argon-YAG laser iridotomies in dark irides. $\mathrm{Br}$ J Ophthalmol. 1992;76(6):329-331.

32. Buckley SA, Reeves B, Burdon M, et al. Acute angle closure glaucoma: relative failure of YAG iridotomy in affected eyes and factors influencing outcome. Br J Ophthalmol. 1994;78(7):529-533.

33. Fleck BW, Wright E, Fairley EA. A randomised prospective comparison of operative peripheral iridectomy and Nd:YAG laser iridotomy treatment of acute angle closure glaucoma: 3 year visual acuity and intraocular pressure control outcome. Br J Ophthalmol. 1997;81(10):884-888. 
34. Choi JS, Kim YY. Progression of peripheral anterior synechiae after laser iridotomy. Am J Ophthalmol. 2005;140(6):1125-1127.

35. Spaeth GL, Idowu O, Seligsohn A, et al. The effects of iridotomy size and position on symptoms following laser peripheral iridotomy. J Glaucoma. 2005;14(5):364-367.

36. Murphy PH, Trope GE. Monocular blurring. A complication of YAG laser iridotomy. Ophthalmology. 1991;98(10):1539-1542.

37. Hodes BL, Bentivegna JF, Weyer NJ. Hyphema complicating laser iridotomy. Arch Ophthalmol. 1982;100(6):924-925.

38. Krupin T, Stone RA, Cohen BH, Kolker AE, Kass MA. Acute intraocular pressure response to argon laser iridotomy. Ophthalmology. 1985;92(7):922-926.

39. Tarongoy P, Ho CL, Walton DS. Angle-closure glaucoma: the role of the lens in the pathogenesis, prevention, and treatment. Surv Ophthalmol. 2009;54(2):211-225.

40. Tan GS, Hoh ST, Husain R, et al. Visual acuity after acute primary angle closure and considerations for primary lens extraction. Br JOphthalmol. 2006;90(1):14-16.

41. Nonaka A, Kondo T, Kikuchi M, et al. Angle widening and alteration of ciliary process configuration after cataract surgery for primary angle closure. Ophthalmology. 2006;113(3):437-441.

42. Jacobi PC, Dietlein TS, Lüke C, Engels B, Krieglstrein GK. Primary phacoemulsification and intraocular lens implantation for acute angleclosure glaucoma. Ophthalmology. 2002;109(9):1597-1603.

43. Roberts TV, Francis IC, Lertusumitkul S, Kappagoda MB, Coroneo MT. Primary phacoemulsification for uncontrolled angle-closure glaucoma. J Cataract Refract Surg. 2000;26(7):1012-1016.

44. Zhang Ming Z, Lim AS, Yin Wong T. A pilot study of lens extraction in the management of acute primary angle-closure glaucoma. Am J Ophthalmol. 2003;135(4):534-536.

45. Jacobi PC, Dietlein TS, Lűke C, Engels B, Krieglstein GK. Primary phacoemulsification and intraocular lens implantation for acute angle-closure glaucoma. Ophthalmology. 2002;109(9): $1597-1603$

46. Lam DS, Leung DY, Tham CC, et al. Randomized trial of early phacoemulsification versus peripheral iridotomy to prevent intraocular pressure rise after acute primary angle closure. Ophthalmology. 2008; 115(7):1134-1140.

47. Varma D, Baylis O, Wride N, Phelan PS, Fraser SG. Viscogonioplasty: an effective procedure for lowering intraocular pressure in primary angle closure glaucoma. Eye (Lond). 2007;21(4):472-475.

48. Lai JS, Tham CC, Lam, DS. Incisional surgery for angle closure glaucoma. Semin Ophthalmol. 2002;17(2):92-99.

49. Imaizumi M, Takaki $Y$, Yamashita H. Phacoemulsification and intraocular lens implantation for acute angle closure not treated or previously treated by laser iridotomy. J Cataract Refract Surg. 2006; 32(1):85-90.

50. Lai JS, Tham CC, Chan JS. The clinical outcomes of cataract extraction by phacoemulsification in eyes with primary angle-closure glaucoma (PACG) and co-existing cataract: a prospective case series. J Glaucoma. 2006; 15(1):47-52.

51. Campbell DG, Vela, A. Modern goniosynechialysis for the treatment of synechial angle-closure glaucoma. Ophthalmology. 1984;91(9): 1052-1060
52. Shingleton BJ, Chang, MA, Bellows AR, Thomas JV. Surgical goniosynechialysis for angle-closure glaucoma. Ophthalmology. 1990; 97(5):551-556

53. Tanihara H, Nagata M. Argon-laser gonioplasty following goniosynechialysis. Graefe's Arch Clin Exp Ophthalmol. 1991;229(6): 505-507.

54. Teekhasaenee C, Ritch R. Combined phacoemulsification and goniosynechialysis for uncontrolled chronic angle-closure glaucoma after acute angle-closure glaucoma. Ophthalmology. 1999;106(4):669-675.

55. Harasymowycz PJ,Papamatheakis DG,Ahmed I, etal. Phacoemulsification and goniosynechialysis in the management of unresponsive primary angle closure. J Glaucoma. 2005;14(3):186-189.

56. Lai JS, Tham CC, Lam, DS. The efficacy and safety of combined phacoemulsification, intraocular lens implantation, and limited goniosynechialysis, followed by diode laser peripheral iridoplasty, in the treatment of cataract and chronic angle-closure glaucoma. J Glaucoma. 2001;10(4):309-315.

57. Lai JS, Tham CC, Chua JK, Lam DS. Immediate diode laser peripheral iridoplasty as treatment of acute attack of primary angle closure glaucoma: a preliminary study. J Glaucoma. 2001;10(2):89-94.

58. Lai JS, Tham CC, Chua JK, Lam DS. Efficacy and safety of inferior 180 degrees goniosynechialysis followed by diode laser peripheral iridoplasty in the treatment of chronic angle-closure glaucoma. J Glaucoma. 2000;9(5):388-391.

59. Razeghinejad MR. Combined phacoemulsification and viscogoniosynechialysis in patients with refractory acute angle-closure glaucoma. J Cataract Refract Surg. 2008;34(5):827-830.

60. Bellucci R, Perfetti S, Babighian S, Morselli S, Bonomi L. Filtration and complications after trabeculectomy and after phaco-trabeculectomy. Acta Ophthalmol Scand Suppl. 1997;75(224):44-45.

61. Aung T, Tow SL, Yap EY, Chan SP, Seah SK. Trabeculectomy for acute primary angle closure. Ophthalmology. 2000;107(7):1298-1302.

62. Membrey WL, Poinoosawmy DP, Bunce C, Hitchings RA. Glaucoma surgery with or without adjunctive antiproliferatives in normal tension glaucoma: 1 intraocular pressure control and complications. $\mathrm{Br} J$ Ophthalmol. 2000;84(6):586-590.

63. Costa VP, Smith M, Spaeth GL, Gandham S, Markovitz B. Loss of visual acuity after trabeculectomy. Ophthalmology. 1993;100(5):599-612.

64. Yoon PS, Singh K. Update on antifibrotic use in glaucoma surgery, including use in trabeculectomy and glaucoma drainage implants and combined cataract and glaucoma surgery. Curr Opin Ophthalmol. 2004; 15(2):141-146.

65. Budenz DL, Pyfer M, Singh K, Gordon J, Piltz-Seymour J, Keates EU. Comparison of phacotrabeculectomy with 5-fluorouracil, mitomycinC, and without antifibrotic agents. Ophthalmic Surg Lasers. 1999; 30(5):367-374.

66. Bindlish R, Condon GP, Schlosser JD, D'Antonio J, Lauer KB, Lehrer R. Efficacy and safety of mitomycin-C in primary trabeculectomy: fiveyear follow-up. Ophthalmology. 2002;109(7):1336-1341; discussion 1341-1332.

67. Poulsen EJ, Allingham RR. Characteristics and risk factors of infections after glaucoma filtering surgery. J Glaucoma. 2000;9(6):438-443.

68. Khaw PT, Migdal CS. Current techniques in wound healing modulation in glaucoma surgery. Curr Opin Ophthalmol. 1996;7(2):24-33.
Clinical Ophthalmology

\section{Publish your work in this journal}

Clinical Ophthalmology is an international, peer-reviewed journal covering all subspecialties within ophthalmology. Key topics include: Optometry; Visual science; Pharmacology and drug therapy in eye diseases; Basic Sciences; Primary and Secondary eye care; Patient Safety and Quality of Care Improvements. This journal is indexed on Submit your manuscript here: http://www.dovepress.com/clinical-ophthalmology-journal

\section{Dovepress}

PubMed Central and CAS, and is the official journal of The Society of Clinical Ophthalmology (SCO). The manuscript management system is completely online and includes a very quick and fair peer-review system, which is all easy to use. Visit http://www.dovepress.com/ testimonials.php to read real quotes from published authors. 